\title{
Dual fluorescence and intramolecular charge transfer with crystalline 4-(diisopropylamino)benzonitrile
}

\author{
Sergey I. Druzhinin ${ }^{1}$, Attila Demeter ${ }^{2}$, Klaas A. Zachariasse * \\ Max-Planck-Institut für Biophysikalische Chemie, Spektroskopie und Photochemische Kinetik, 37070 Göttingen, Germany
}

Received 8 August 2001

\begin{abstract}
Dual fluorescence from a locally excited (LE) and an intramolecular charge transfer (ICT) state is observed with 4-(diisopropylamino)benzonitrile (DIABN) crystals, from 80 down to $-110{ }^{\circ} \mathrm{C}$. With crystalline 4-(methylamino)benzonitrile (MABN) only LE emission is observed over this temperature range. Crystals of 4-(dimethylamino)benzonitrile (DMABN) at room temperature mainly show LE fluorescence, whereas the red-shifted structured phosphorescence increases in importance upon cooling. Its spectral shape resembles that of DMABN phosphorescence in low-temperature glassy media, red-shifted by around $2800 \mathrm{~cm}^{-1}$. The ICT fluorescence of DIABN crystals at -110 ${ }^{\circ} \mathrm{C}$ has a risetime of $55 \mathrm{ps}$, which becomes shorter with increasing temperature. (C) 2001 Elsevier Science B.V. All rights reserved.
\end{abstract}

\section{Introduction}

4-(Diisopropylamino)benzonitrile (DIABN) emits dual fluorescence as a result of intramolecular charge transfer (ICT) in nonpolar solvents such as alkanes [1] as well as in the vapour phase [2], in contrast to 4-(dimethylamino)benzonitrile (DMABN), for which only fluorescence from the locally excited (LE) $S_{1}$ state is found under these conditions [2-6]. In the case of 4-(methylamino)benzonitrile (MABN), dual fluorescence is not observed, even not in strongly polar solvents

\footnotetext{
*Corresponding author. Fax: +49-551-201-1501.

E-mail address: kzachar@gwdg.de (K.A. Zachariasse).

${ }^{1}$ Permanent address: Department of Chemistry, Moscow State University, Moscow 119899, Russia.

${ }^{2}$ On leave from the Institute of Chemistry, Chemical Research Center, Hungarian Academy of Sciences, P.O. Box 17, 1525 Budapest, Hungary.
}

such as acetonitrile or formamide at low temperatures $[7,8]$.

The appearance of ICT and dual fluorescence with DIABN in alkane solvents has been attributed to its relatively small energy gap $\Delta E\left(\mathrm{~S}_{1}, \mathrm{~S}_{2}\right)$ between the two lowest excited singlet states, considerably smaller than that of DMABN [1], which leads to a lowering of the barrier for the ICT reaction [1]. The observation of dual fluorescence with DIABN in the vapour phase [2] shows that an interaction with nonpolar or polar solvent molecules is not an essential requirement for the occurrence of an ICT reaction in aminobenzonitriles. DIABN was therefore considered to be a good candidate for an investigation of ICT reactions in the crystalline state.

It follows from X-ray analysis [1,9] that the DIABN molecules do not occur as sandwich pairs in the crystal, but are arranged in a head-to-head $\mathrm{T}$-shaped configuration, similar to what is found 
for DMABN, except that for this molecule the pairs are arranged head-to-tail [10,11]. DMABN crystals undergo a solid-solid phase transition upon cooling $[10,11]$. Such a phase transition is not observed with crystalline DIABN, down to $130 \mathrm{~K}$ [9].

In the fluorescence spectrum at $20{ }^{\circ} \mathrm{C}$ of DMABN deposited as a solid film by solvent evaporation [12], two structureless emission bands have been observed. The band to the red of the LE fluorescence is similar in shape and spectral position to that reported for DMABN aggregates, which are described as head-to-tail sandwich dimers [12]. For crystalline DMABN [11], however, the fluorescence spectrum at room temperature mainly consists of an LE emission.

Structural changes taking place in powdered crystalline DMABN at $25^{\circ} \mathrm{C}$ after picosecond laser excitation have recently been investigated, employing X-ray diffraction with a time resolution of $60 \mathrm{ps}$ [13]. It was shown that upon excitation to the $\mathrm{S}_{1}(\mathrm{LE})$ state, the averaged torsional angle $\theta$ of the dimethylamino group relative to the plane of the phenyl ring increases from 0 to $10^{\circ}$. In addition, a reduction of the pyramidality of the amino nitrogen was found to occur during the excited state lifetime, the averaged inversion angle changing from $13^{\circ}$ in $\mathrm{S}_{0}$ to $3^{\circ}$ in $\mathrm{S}_{1}$. DMABN does not show appreciable dual fluorescence in the crystalline state at room temperature [11], nor at this temperature in weakly polar solvents such as toluene [6] and diethyl ether [14], having effective polarities similar to those prevailing in crystalline aromatic amines. The relatively small twist of the amino group occurring in the singlet excited state of crystalline DMABN [13] can therefore not be attributed to an ICT reaction resulting in a twisted intramolecular charge transfer (TICT) state [15], for which a full twist over $90^{\circ}$ and electronic decoupling (proportional to $\cos ^{2} \theta$ [16]) of the amino and benzonitrile moieties is required [4].

In the present paper an investigation of the photophysics of crystalline DIABN is reported. For comparison, DMABN and MABN crystals were also studied. For the last molecule, as mentioned above, dual fluorescence has not been observed under any condition of solvent polarity or temperature.

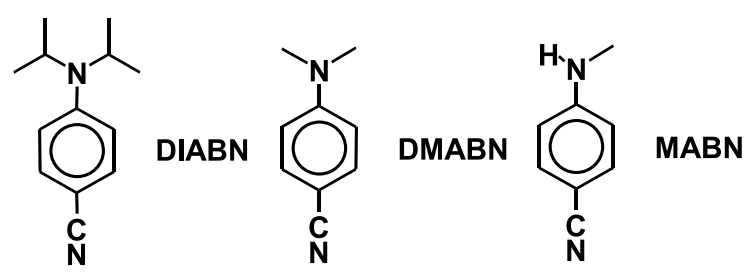

\section{Experimental}

The synthesis of DIABN [1] and of MABN [6] was described previously. DMABN, obtained from Aldrich, was sublimed before use. The crystals were grown from cyclopentane or from an $n$-hexane/diethyl ether mixture. The fluorescence spectra of the crystals, contained in a quartz tube purged with nitrogen, were measured with a quantum-corrected ISA-Spex Fluorolog 3-22 or a Shimadzu RF-5000PC spectrofluorometer. The fluorescence decay times were determined with picosecond laser single-photon counting (SPC) setups. These setups and the analysis procedure of the fluorescence decays have been described previously [6,17]. The instrument response function has a half-width of $20-35$ ps and the time resolution is estimated at $3-5$ ps [1].

\section{Results and discussion}

\subsection{Crystal fluorescence spectra at room tempera- ture}

The fluorescence spectrum of crystalline MABN at $25{ }^{\circ} \mathrm{C}$ (Fig. 1a) consists of an emission band with a shape similar to that measured in solution [16]. Its spectral position is red-shifted by approximately $1300 \mathrm{~cm}^{-1}$ as compared with that in diethyl ether [16]. From the absence of dual fluorescence it is concluded that the crystal fluorescence of MABN originates from an LE state. For DMABN crystals (Fig. 1b), an emission spectrum with a maximum at $26000 \mathrm{~cm}^{-1}$ and a relatively weak additional red-shifted band is observed, in agreement with the spectrum of powdered DMABN crystals at room temperature reported in the literature [11]. 
$\lambda[\mathrm{nm}]$

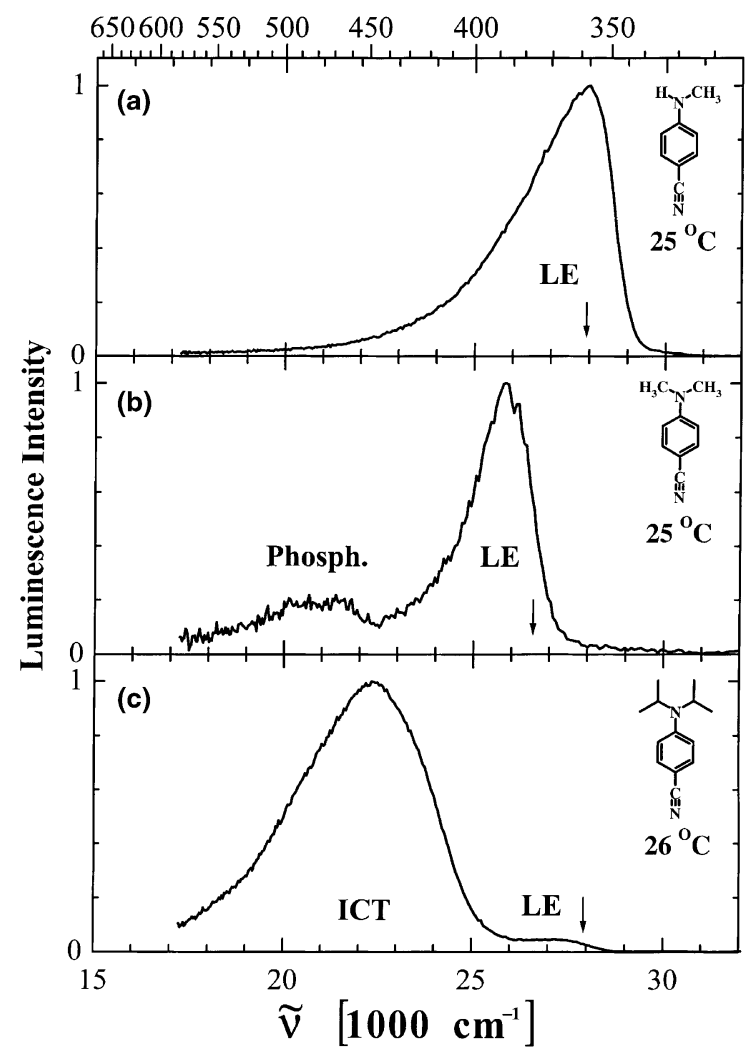

Fig. 1. Luminescence spectra at around $25^{\circ} \mathrm{C}$ of crystalline (a) 4-(methylamino)benzonitrile (MABN), (b) 4-(dimethylamino)benzonitrile (DMABN) and (c) 4-(diisopropylamino)benzonitrile (DIABN). The fluorescence of MABN (a) originates from a locally excited (LE) state, whereas with DMABN (b) a sphosphorescence appears to the red of the LE emission. The fluorescence of DIABN consists of emissions from a locally excited (LE) and an intramolecular charge transfer (ICT) state. The arrows indicate the onset of the crystal absorption. Excitation wavelength: $300 \mathrm{~nm}$.

With crystals of DIABN, the fluorescence spectrum at $26^{\circ} \mathrm{C}$ consists of two emission bands, see Fig. 1c. The emission around $27500 \mathrm{~cm}^{-1}$, in the spectral range of the MABN crystal fluorescence (Fig. 1a), is assumed to come from an LE state. The main broad emission band with a maximum at $22300 \mathrm{~cm}^{-1}$ is comparable in shape with the intramolecular charge transfer (ICT) band of DIABN in solvents such as $n$-hexane and diethyl ether [1] and is hence identified as ICT fluorescence of crystalline DIABN.

\subsection{Fluorescence spectra as a function of temper-} ature

The fluorescence spectrum of crystalline DIABN at $-110{ }^{\circ} \mathrm{C}$ (Fig. 2a) is similar to that at $26{ }^{\circ} \mathrm{C}$ (Fig. 1c), with an ICT band and a smaller LE emission. The ICT/LE fluorescence intensity ratio, gradually decreasing upon lowering the temperature, is clearly smaller at $-110{ }^{\circ} \mathrm{C}$ as compared with $26^{\circ} \mathrm{C}$. This could be due to a decrease of the rate constant $k_{\mathrm{a}}$ of the LE $\rightarrow$ ICT

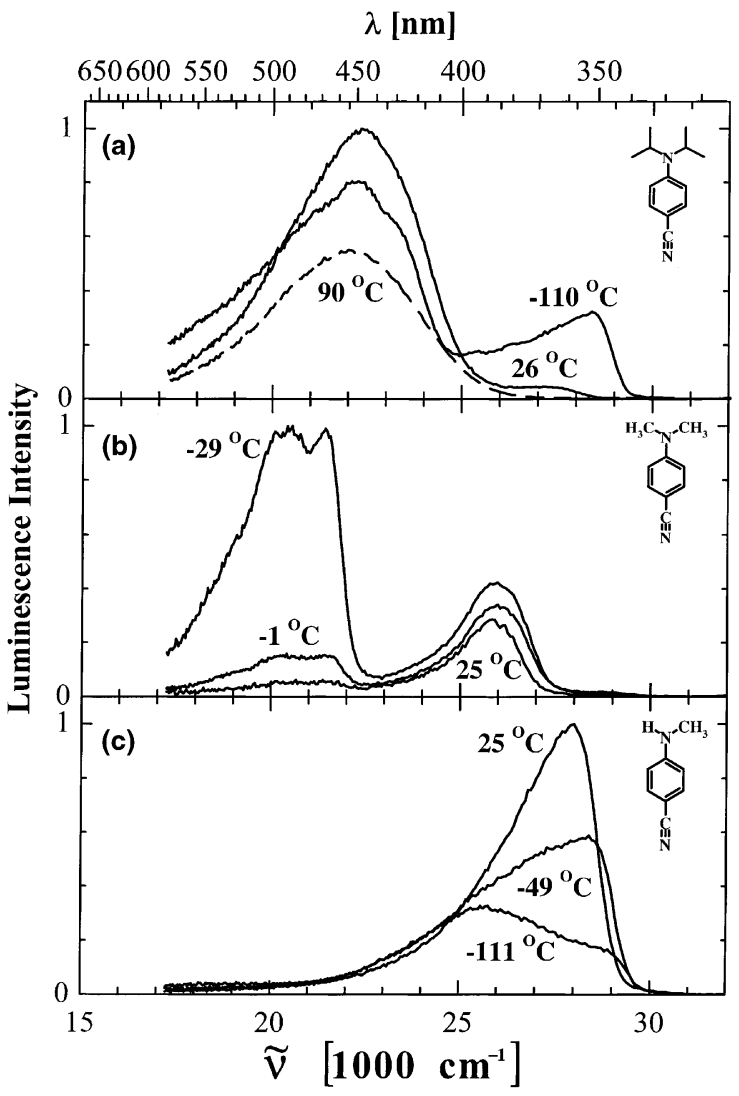

Fig. 2. Luminescence spectra at various temperatures of crystalline (a) 4-(diisopropylamino)benzonitrile (DIABN), (b) 4-(dimethylamino)benzonitrile (DMABN) and (c) 4-(methylamino)benzonitrile (MABN). The fluorescence of DIABN (a) consists of emissions from a locally excited (LE) and an intramolecular charge transfer (ICT) state. The dashed spectrum in (a) is from molten DIABN at $90{ }^{\circ} \mathrm{C}$, see text. With DMABN (b) phosphorescence of the LE emission, whereas the fluorescence of MABN (c) originates from an LE state. Excitation wavelength: $300 \mathrm{~nm}$. See Fig. 1. 
reaction upon cooling [1], as in the low-temperature limit $\left(k_{\mathrm{d}} \ll 1 / \tau_{0}^{\prime}\right)$ of the ICT/LE fluorescence quantum yield ratio $\Phi^{\prime}(\mathrm{ICT}) / \Phi(\mathrm{LE})$, see Eq. (1), this ratio becomes equal to $k_{\mathrm{f}}^{\prime} / k_{\mathrm{f}} \cdot k_{\mathrm{a}} \tau_{0}^{\prime}$ and hence decreases with decreasing temperature $[18,19]$

$\Phi^{\prime}(\mathrm{ICT}) / \Phi(\mathrm{LE})=k_{\mathrm{f}}^{\prime} / k_{\mathrm{f}} \cdot k_{\mathrm{a}} /\left(k_{\mathrm{d}}+1 / \tau_{0}^{\prime}\right)$.

In Eq. (1), $k_{\mathrm{f}}^{\prime}$ and $k_{\mathrm{f}}$ are the ICT and LE radiative rate constants, $k_{\mathrm{d}}$ is the rate constant for the ICT $\rightarrow$ LE back reaction and $\tau_{0}^{\prime}$ is the ICT fluorescence lifetime.

The fluorescence spectrum of molten DIABN at $90{ }^{\circ} \mathrm{C}$ (Fig. 2a), a few degrees above the crystal melting point $\left(81-84{ }^{\circ} \mathrm{C}\right.$ [1]), consists of a broad ICT band, with no sign of an LE emission. The ICT emission maximum is somewhat red-shifted $\left(300 \mathrm{~cm}^{-1}\right)$ with respect to that of the crystals, possibly a result of the more effective orientational polarization by surrounding molecules in the melt.

In the case of crystalline DMABN (Fig. 2b), the relative as well as the absolute importance of the red-shifted structured emission band increases when the temperature is lowered down to $-29^{\circ} \mathrm{C}$, still above the solid-solid phase transition [11]. The intensity of the red-shifted band further increases upon cooling, reaching at $-110{ }^{\circ} \mathrm{C}$ an intensity ratio of 23 with respect to the fluorescence band at around $26000 \mathrm{~cm}^{-1}$. The shape of the luminescence spectrum of crystalline DMABN resembles that of the fluorescence and phosphorescence of DMABN in low-temperature glassy media [20,21]. Experiments with a mechanical chopper show that the red-shifted band has a long decay time, supporting its identification as phosphorescence. Both bands in the crystal spectrum have undergone a large red-shift of about 3300 and $2800 \mathrm{~cm}^{-1}$, respectively. Also the excitation spectrum, similar for the two bands in the crystal luminescence, is red-shifted by a comparable amount, see Fig. 2b. Based on the observations reported here, it is concluded that crystalline DMABN does not emit appreciable ICT fluorescence.

The fluorescence spectrum of MABN crystals changes in shape upon cooling, see Fig. 2c, the blue part of the emission band decreasing in intensity from 25 to $-11{ }^{\circ} \mathrm{C}$. This decrease cannot be caused by a change in the reabsorption of the emission by the crystal (large optical density), as the onset of the excitation spectrum at at $25{ }^{\circ} \mathrm{C}$ only starts at $28600 \mathrm{~cm}^{-1}$, as indicated by an arrow in Fig. 1a, and a blue-shift of this spectrum by around $900 \mathrm{~cm}^{-1}$ takes place when lowering the temperature to $-110^{\circ} \mathrm{C}$. Note that the position of the fluorescence maximum at $-111^{\circ} \mathrm{C}$ is similar to that of DMABN crystals (Fig. 2b). There clearly is no spectral indication for the appearance of dual fluorescence involving an ICT state for crystalline MABN at these temperatures.

\subsection{LE and ICT fluorescence decays of crystalline $D I A B N$ at $-110^{\circ} \mathrm{C}$}

The fluorescence decay of crystalline DIABN was measured as a function of temperature, from 25 to $-110{ }^{\circ} \mathrm{C}$, at several wavelengths spanning the emission spectrum. The global analysis of the decays $i_{\mathrm{f}}(\mathrm{LE})$ and $i_{\mathrm{f}}(\mathrm{ICT})$ at $-110{ }^{\circ} \mathrm{C}$ is shown in Fig. 3a. The LE decay is practically single exponential, with a main $(97 \%$ contribution, $\left.A_{12} \tau_{2} / \Sigma_{i} A_{1 i} \tau_{i}\right)$ short decay time $\tau_{2}$ of $55 \mathrm{ps}$ and a weak $(3 \%)$ longer decay time $\tau_{1}$ of $2.79 \mathrm{~ns}$.

$$
\begin{aligned}
& i_{\mathrm{f}}(\mathrm{LE})=A_{11} \exp \left(-t / \tau_{1}\right)+A_{12} \exp \left(-t / \tau_{2}\right), \\
& i_{\mathrm{f}}(\mathrm{ICT})=A_{21} \exp \left(-t / \tau_{1}\right)+A_{22} \exp \left(-t / \tau_{2}\right), \\
& A=A_{12} / A_{11} \sim k_{\mathrm{a}} / k_{\mathrm{d}}
\end{aligned}
$$

(low-temperature limit : $\left.k_{\mathrm{d}} \ll 1 / \tau_{0}^{\prime}[17]\right)$.

The relatively large amplitude ratio $\left(A_{12} / A_{11}=\right.$ 1970), see Eq. (2), indicates that the forward ICT reaction rate constant $k_{\mathrm{a}}$ is about 2000 times larger than the thermal back reaction $k_{\mathrm{d}}$, as $k_{\mathrm{a}} / k_{\mathrm{d}}$ is roughly equal to $A_{12} / A_{11}$, see Eq. (4) [17]. This is not unreasonable, as with DIABN in $n$-hexane solution at $-90^{\circ} \mathrm{C} k_{\mathrm{d}}$ is likewise much smaller than $k_{\mathrm{a}}$, as follows from the amplitude ratio $A_{12} / A_{11}$ of 211 obtained at this temperature [1].

The ICT fluorescence, measured at the emission maximum (473 nm, Fig. 2a), shows a growing-in with a time $\tau_{2}$ of $55 \mathrm{ps}$, the same as the main LE decay time at $378 \mathrm{~nm}$. The time $\tau_{1}(2.79 \mathrm{~ns})$ is equal to the ICT lifetime $\tau_{0}^{\prime}$ [1]. The amplitude ratio $A_{22} / A_{21}$ of -1.04 obtained for the ICT fluorescence decay from the global analysis in Fig. 3a, means that this ICT state is not formed by direct excita- 


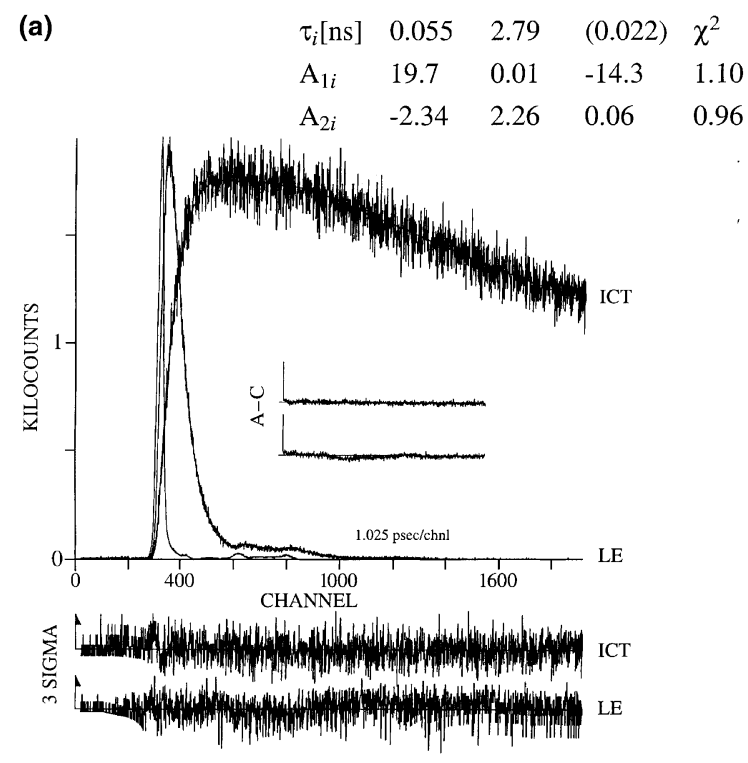

(b) $\quad \begin{array}{lllllll}\tau_{i}(\mathrm{~ns}) & 0.011 & 2.92 & (0.044) & (0.444) & \chi^{2}\end{array}$

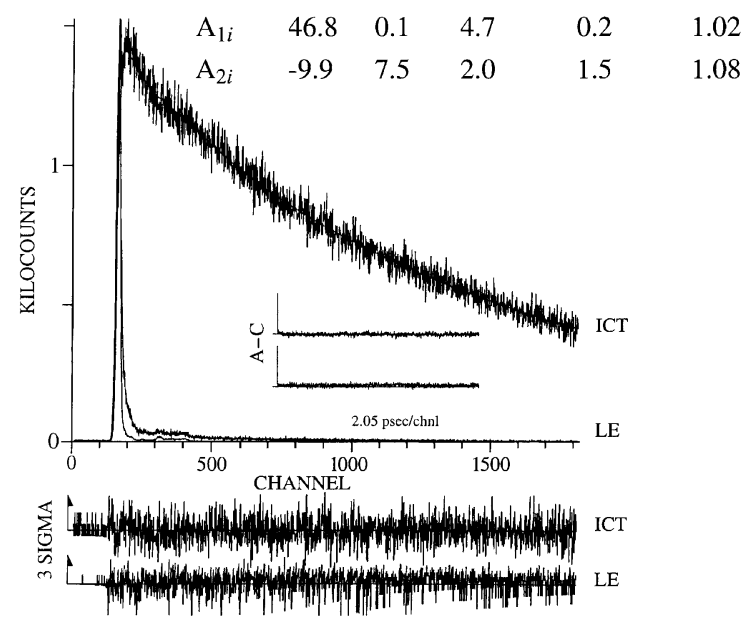

Fig. 3. LE and ICT fluorescence response functions of crystalline 4-(diisopropylamino)benzonitrile (DIABN) at (a) -110 ${ }^{\circ} \mathrm{C}$ and (b) $25^{\circ} \mathrm{C}$. The LE and ICT decays are analysed simultaneously (global analysis). The decay times $\left(\tau_{2}, \tau_{1}\right)$ and their preexponential factors $A_{1 i}$ and $A_{2 i}$ are given (Eqs. (2) and (3)). The shortest decay time $\tau_{2}$ is listed first. The times in parentheses are attributed to crystal defects (see text). The weighted deviations, expressed in $\sigma$ (expected deviations), the autocorrelation functions $\mathrm{A}-\mathrm{C}$, and the values for $\chi^{2}$ are also indicated. Excitation wavelength: $328 \mathrm{~nm}$. Emission wavelength: $378 \mathrm{~nm}$ (a) and $373 \mathrm{~nm}$ (b) for LE; $473 \mathrm{~nm}$ for ICT. tion from the ground state, i.e., LE is the precursor of the ICT state in the DIABN crystal fluorescence $[1,17]$.

The short time of 22 ps resulting from the global analysis of the LE and ICT decays in Fig. 3a is attributed to specific crystal effects, such as defects and other packing disorders. Similar short times are found in the LE fluorescence decay of DMABN and MABN, see Figs. 4 and 5.

\subsection{DIABN crystal fluorescence decays at $26{ }^{\circ} \mathrm{C}$}

The LE and ICT fluorescence decays of a DIABN crystal at $26{ }^{\circ} \mathrm{C}$ are shown in Fig. 3b, in a global fit with four exponentials. The LE decay has a short decay time of 11 ps $(47 \%)$ and a long time of $2.92 \mathrm{~ns}(26 \%)$. In the ICT decay, a risetime of 11 ps appears (global analysis, Fig. 3b) and the long decay time of $2.92 \mathrm{~ns}$ represents the lifetime $\tau_{0}^{\prime}$ of the ICT state. The times of $44 \mathrm{ps}$ and $0.444 \mathrm{~ns}$ are attributed to specific crystal effects and are therefore discarded, see previous section. In the ICT fluorescence response function of crystalline DIABN excited at $276 \mathrm{~nm}$, a decay pattern similar to that of Fig. $3 \mathrm{~b}$ is observed, although a risetime could not be resolved.

\subsection{MABN crystal fluorescence decays at -110 and $25^{\circ} \mathrm{C}$}

The fluorescence decay of crystalline MABN at $-110{ }^{\circ} \mathrm{C}$, measured at the maximum of the LE emission at this temperature (Fig. 2c), is double exponential, with a main decay time $\tau_{1}$ of $3.42 \mathrm{~ns}$ (99 percent) and a shorter time $\tau_{2}$ of $0.495 \mathrm{~ns}(1 \%)$, see Fig. 4a. At $25{ }^{\circ} \mathrm{C}$ (Fig. 4b) a more complex decay is found, with a long decay time of $2.59 \mathrm{~ns}$ $(69 \%)$ and two shorter times: $0.85 \mathrm{~ns}(25 \%)$ and 0.18 ns $(6 \%)$. As the crystal fluorescence spectrum of MABN gives no indication for dual fluorescence, the complexity of the decays is again (Sections 3.3 and 3.4) attributed to crystal structure effects. This interpretation is supported by the observation that the relative amplitudes (Eq. (2)) of the shorter decay times increase upon prolonged laser excitation and depend on the crystallization procedure. 
(a)
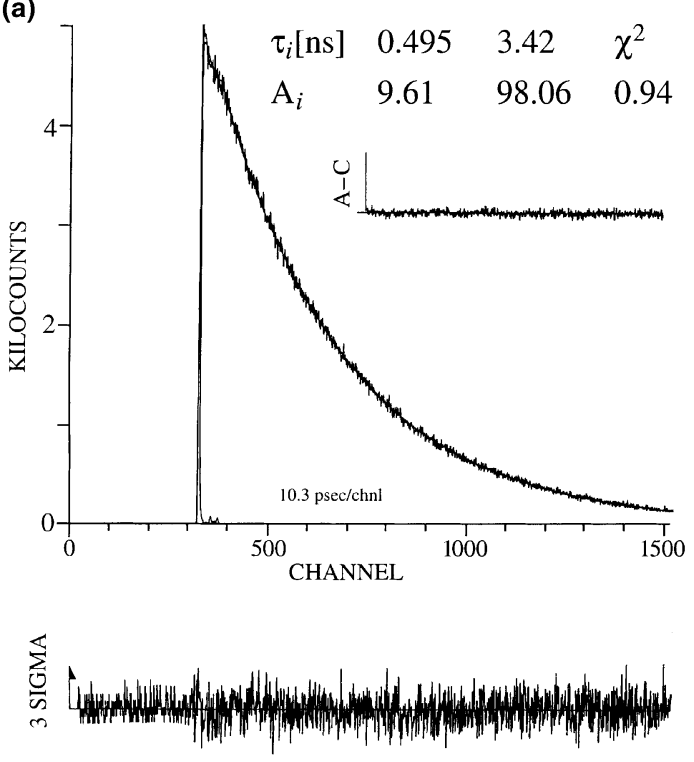

(b)
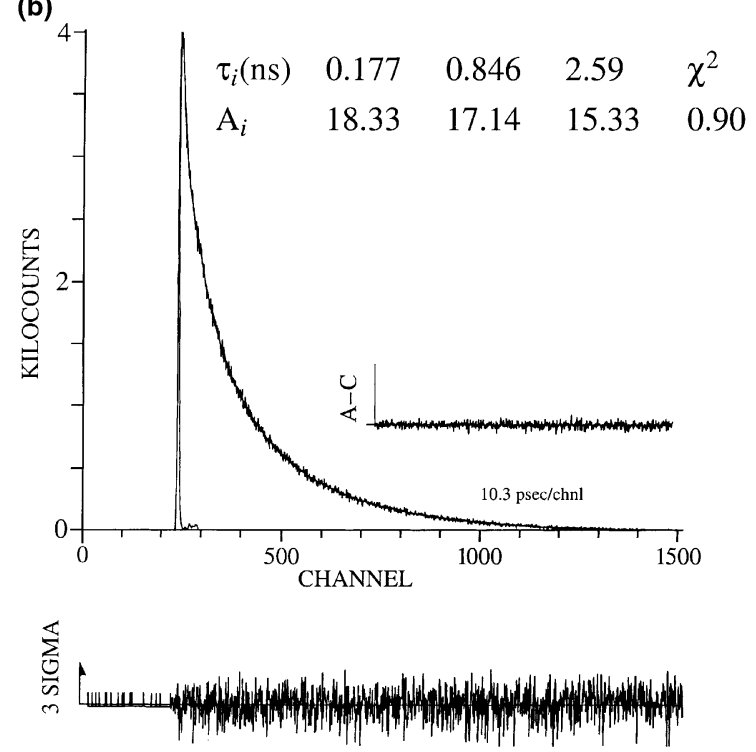

Fig. 4. LE fluorescence response functions of crystalline 4(methylamino)benzonitrile (MABN) at (a) $-110{ }^{\circ} \mathrm{C}$ and (b) $25{ }^{\circ} \mathrm{C}$. The decay times $\tau_{i}$ and their preexponential factors $A_{1 i}$ are given (Eq. (2)). The time in parentheses is attributed to crystal defects (see text). Excitation wavelength: $333 \mathrm{~nm}$ (a) and $293 \mathrm{~nm}$ (b). Emission wavelength: $383 \mathrm{~nm}$ (a) and $353 \mathrm{~nm}$ (b). See the caption of Fig. 3.
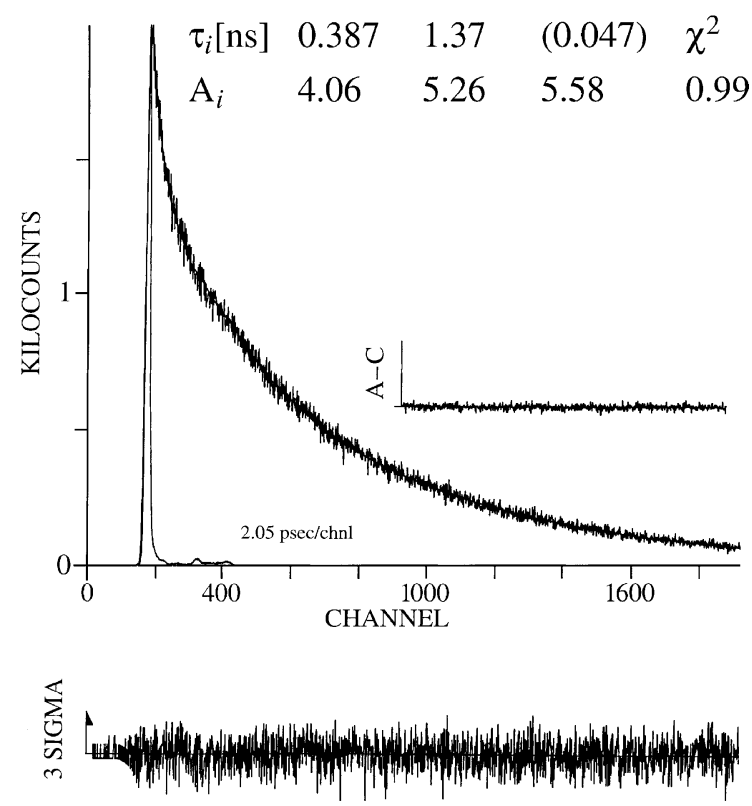

Fig. 5. LE fluorescence response functions of crystalline 4(dimethylamino)benzonitrile (DMABN) at $25{ }^{\circ} \mathrm{C}$. The decay times $\tau_{i}$ and their preexponential factors $A_{1 \mathrm{i}}$ are given (Eq. (2)). The time in parentheses is attributed to crystal defects (see text). Excitation wavelength: $328 \mathrm{~nm}$. Emission wavelength: $383 \mathrm{~nm}$. See the caption of Fig. 3.

\subsection{DMABN crystal fluorescence decays at $25^{\circ} \mathrm{C}$}

The fluorescence decay of crystalline DMABN at $25^{\circ} \mathrm{C}$ (Fig. 5), measured near the maximum of the LE emission (383 nm, see Fig. 1b) is triple exponential, with a main ( $80 \%$ ) decay time $\tau_{1}$ of 1.37 ns. The decay time $\tau_{2}$ of 387 ps contributes $17 \%$ to the total decay, whereas the shortest time $\tau_{3}$ (47 ps) is of smaller importance $(3 \%)$. This last time is considered to originate from the quenching effect of intrinsic crystalline defects and irregularities, as also mentioned in previous sections for DIABN and MABN crystals. The times $\tau_{1}(1.37 \mathrm{~ns})$ and $\tau_{2}$ (387 ps) observed here, are in reasonable agreement with the decay times reported for powdered crystalline DMABN (1.71 and $0.53 \mathrm{~ns}$ ) [13].

3.7. Crystal packing of DIABN, DMABN and $M A B N$. Pair separation

In the crystal of DIABN, the molecules are present as pairs in a T-shaped configuration, with 
a head-to-head arrangement [9-11]. In crystalline MABN, the molecules are likewise arranged headto-head, whereas a head-to-tail orientation is observed for DMABN crystals $[10,11]$. In crystalline MABN, the molecules are packed in stacks, with an intermolecular distance of $6.27 \AA$ between the centres of the phenyl groups [9], whereas this distance is $5.05 \AA$ for DIABN [9] and $4.75 \AA$ for DMABN [9-11]. For the distance between the edge of one phenyl ring to the plane of the other in the two neighbouring DIABN molecules (Tshaped configuration) a value of $4.6 \AA$ is obtained. This separation is considerably larger than the interplanar distance of $3.55 \AA$ observed for the slightly shifted sandwich dimers in pyrene crystals [22], which means that intermolecular interaction between the two molecules in the aminobenzonitrile pairs is not expected to strongly influence their photophysical behaviour. These results explain the absence of dimer emission from the crystals of the three aminobenzonitriles. DIABN crystals do not undergo solid-solid phase transitions down to $-143{ }^{\circ} \mathrm{C}$, in contrast to DMABN, for which a phase transition occurs at around -40 ${ }^{\circ} \mathrm{C}[11,12]$.

\subsection{ICT emission maximum. Crystal polarity}

From a comparison of the ICT emission band of crystalline DIABN at $26{ }^{\circ} \mathrm{C}$ (Fig. 2a) with that of DIABN in solution at this temperature [1], it follows that the fluorescence maximum $\tilde{v}^{\max }$ (ICT) at $22300 \mathrm{~cm}^{-1}$ of the crystal corresponds to that of a solution of ethyl acetate $\left(\varepsilon^{25}=6.0\right)$ at 22300 $\mathrm{cm}^{-1}$ and tetrahydrofuran $\left(\varepsilon^{25}=7.4\right)$ at 22500 $\mathrm{cm}^{-1}$ [1]. It is concluded from this observation that the DIABN crystal has an effective polarity similar to these two solvents. For a melt of DIABN at $90{ }^{\circ} \mathrm{C}$ (Fig. 2a), $\tilde{v}^{\max }(\mathrm{ICT})$ at 22000 $\mathrm{cm}^{-1}$ has a lower value than in the crystal, indicating an increase in polarity upon melting, see Section 3.2.

\section{Conclusions}

DIABN emits dual fluorescence in the crystalline state between 80 and $-110{ }^{\circ} \mathrm{C}$. The predomi- nant red-shifted broad emission band originates from an ICT state, as concluded from a comparison with the ICT fluorescence spectra of DIABN in a series of solvents as a function of polarity. The energy of the emission maximum in the crystal has a value similar to that of ethyl acetate and tetrahydrofuran solutions. With crystalline MABN only LE fluorescence is observed. In the case of DMABN crystals, a second emission appears, redshifted with respect to the LE fluorescence. This new structured emission, strongly increasing in absolute and relative intensity upon cooling, is attributed to phosphorescence and evidence for an ICT fluorescence is not found. The ICT emission of DIABN crystals has a risetime of 55 ps at -110 ${ }^{\circ} \mathrm{C}$, which time decreases with increasing temperature. The same decay times (55 ps and 2.79 ns at $-110{ }^{\circ} \mathrm{C}$ ) are obtained for the LE and ICT emissions, with approximately equal preexponential factors for the ICT fluorescence decay, which shows that the ICT state is not accessible by direct excitation of the ground state of crystalline DIABN. The fluorescence decays of crystalline MABN and DMABN are double or triple exponential, without growing-in components.

The observation of dual fluorescence for DIABN, having a $\mathrm{T}$-shaped pair arrangement in the crystal, shows that the interaction with solvent molecules is not needed for the appearance of ICT in aminobenzonitriles. This can also be concluded from the observation of ICT emission with DIABN in the vapour phase. As crystalline DIABN shows strong ICT fluorescence, it is a promising candidate for a picosecond X-ray analysis such as reported for the LE state of DMABN in [13]. From such an experiment, the molecular structure of the ICT state of DIABN can be determined in a direct manner, in particular the change in twist angle between the amino and the benzonitrile moieties taking place during the $\mathrm{LE} \rightarrow \mathrm{ICT}$ reaction.

\section{Acknowledgements}

The generous support of the Volkswagen Foundation (Project Intra- and Intermolecular Electron Transfer) is gratefully acknowledged. 
Many thanks are due to Dr. S. Techert (Göttingen), Dr. H. Port (Stuttgart) and Professor J. Prochorow (Warsaw) for discussions on the photophysics of crystals and to Prof. K. Rotkiewicz (Warsaw) for information on the experiments described in [12]. Professor D. Stalke (Würzburg) and Dr. M. Noltemeyer (Göttingen), see [9], are thanked for information on crystal structure and packing. A.D. acknowledges support of the Hungarian Science Foundation (OTKA Project No. T 33102).

\section{References}

[1] A. Demeter, S. Druzhinin, M. George, E. Haselbach, J.-L. Roulin, K.A. Zachariasse, Chem. Phys. Lett. 323 (2000) 351.

[2] R. Daum, S. Druzhinin, D. Ernst, L. Rupp, J. Schroeder, K.A. Zachariasse, Chem. Phys. Lett. 341 (2001) 272.

[3] E. Lippert, W. Lüder, H. Boos, in: A. Mangini (Ed.), Advances in Molecular Spectroscopy, European Conference on Molecular Spectroscopy, Bologna, Italy, 1959, Pergamon, Oxford, 1962, p. 443.

[4] W. Rettig, Angew. Chem. Int. Ed. Engl. 25 (1986) 971.

[5] K.A. Zachariasse, M. Grobys, E. Tauer, Chem. Phys. Lett. 274 (1997) 372.

[6] W. Schuddeboom, S.A. Jonker, J.M. Warman, U. Leinhos, W. Kühnle, K.A. Zachariasse, J. Phys. Chem. 96 (1992) 10809.
[7] K.A. Zachariasse, Th. von der Haar, A. Hebecker, U. Leinhos, W. Kühnle, Pure Appl. Chem. 65 (1993) 1745.

[8] K.A. Zachariasse, Chem. Phys. Lett. 320 (2000) 8.

[9] D. Stalke, M. Noltemeyer, K.A. Zachariasse, in preparation.

[10] A. Heine, R. Herbst-Irmer, D. Stalke, W. Kühnle, K.A. Zachariasse, Acta Cryst. B 50 (1994) 363.

[11] G.B. Jameson, B.M. Sheikh-Ali, R.G. Weiss, Acta Cryst. B 50 (1994) 703

[12] K. Rotkiewicz, H. Leismann, W. Rettig, J. Photochem. Photobiol. A: Chem. 49 (1989) 347.

[13] S. Techert, F. Schotte, M. Wulff, Phys. Rev. Lett. 86 (2001) 2030.

[14] Th. von der Haar, A. Hebecker, Yu.V. Il'ichev, Y.-B. Jiang, W. Kühnle, K.A. Zachariasse, Recl. Trav. Chim. Pays-Bas 114 (1995) 430.

[15] C. Day, Physics Today 54 (2001) 19.

[16] I. Rückert, A. Hebecker, A.B.J. Parusel, K.A. Zachariasse, Z. Phys. Chem. 214 (2000) 1597, and references cited there.

[17] Yu.V. Il'ichev, W. Kühnle, K.A. Zachariasse, J. Phys. Chem. 102 (1998) 5670.

[18] J.B. Birks, Photophysics of Aromatic Molecules, Wiley, London, 1970.

[19] K.A. Zachariasse, A.L. Maçanita, W. Kühnle, J. Phys. Chem. B 103 (1998) 9356.

[20] M.A. Morgan, G.C. Pimentel, J. Phys. Chem. 93 (1989) 3056.

[21] Th. von der Haar, Ph.D. Thesis, University Göttingen, 1994.

[22] J.B. Birks, A.A. Kazzaz, Proc. Roy. Soc. A 304 (1968) 291. 